\title{
Deterministic Aided STAP for Target Detection in Heterogeneous Situations
}

\author{
J.-F. Degurse, ${ }^{1}$ L. Savy, ${ }^{1}$ S. Marcos, ${ }^{2}$ and J.-Ph. Molinié ${ }^{1}$ \\ ${ }^{1}$ Department of Electromagnetism and Radar, ONERA, 91120 Palaiseau, France \\ ${ }^{2}$ Laboratoire des Signaux et des Systémes, Supéléc-CNRS, University of Paris-Sud, 91192 Gif-sur-Yvette, France \\ Correspondence should be addressed to J.-F. Degurse; jean-francois.degurse@lss.supelec.fr
}

Received 30 April 2013; Accepted 23 September 2013

Academic Editor: Ulrich Nickel

Copyright (c) 2013 J.-F. Degurse et al. This is an open access article distributed under the Creative Commons Attribution License, which permits unrestricted use, distribution, and reproduction in any medium, provided the original work is properly cited.

\begin{abstract}
Classical space-time adaptive processing (STAP) detectors are strongly limited when facing highly heterogeneous environments. Indeed, in this case, representative target free data are no longer available. Single dataset algorithms, such as the MLED algorithm, have proved their efficiency in overcoming this problem by only working on primary data. These methods are based on the APES algorithm which removes the useful signal from the covariance matrix. However, a small part of the clutter signal is also removed from the covariance matrix in this operation. Consequently, a degradation of clutter rejection performance is observed. We propose two algorithms that use deterministic aided STAP to overcome this issue of the single dataset APES method. The results on realistic simulated data and real data show that these methods outperform traditional single dataset methods in detection and in clutter rejection.
\end{abstract}

\section{Introduction}

In the context of radar signal processing, the purpose of space-time adaptive processing (STAP) is to remove ground clutter returns, in order to enhance slow moving target detection. STAP performs two-dimensional space and time adaptive filtering where different space channels are combined at different times [1]. Filter's weights are adaptively computed from training data in the neighborhood of the range cell of interest, called cell under test (CUT). The estimation of these weights is always deducted, more or less directly, from an estimation of the covariance matrix of the received signal, which is the key quantity in the process of adaptation [2]. Any implementation of STAP processing must remain absolutely consistent with the strategy of radar processing whose purpose is to obtain a high probability of detection while keeping a very low probability of false alarm.

Classical space-time adaptive processing (STAP) detectors are strongly limited when facing a severe nonstationary environment such as heterogeneous clutter. Indeed, in these cases, representative training data are no longer available. The Maximum Likelihood Estimation Detector (MLED) [3] is a single dataset detector among others [4]. It only operates with the data from the cell under test, hence its performance is not impacted by nonstationarity. Of course, no environment is purely heterogeneous or homogeneous and the problem can be addressed by combining primary and secondary data [5]. We will here consider the environment to be heterogeneous enough to only use primary data. To make the primary data target-free, the MLED detector removes a thin part of the signal of the Doppler cell under test from the covariance matrix. A slight part of the clutter is removed along the target signal which implies a degradation of clutter rejection, especially if the number of Doppler cells is low. The less Doppler cells, the more the clutter removed from the covariance matrix and the worse the estimation of the covariance matrix. The bad estimation of the matrix can be addressed by using subspace methods [6] but the removal of some clutter is inherent to the APES method.

In this paper, we will show how we can overcome this problem by the use of deterministic aided STAP. Moreover, we will extend this method to the Stop-Band APES which greatly reduces the computational workload of the MLED detector.

Section 2 is devoted to the data model, and Section 3 summarizes the principle of the MLED APES-based detector and the Stop-Band APES algorithm. A deterministic based nonadaptive approach of space-time processing is presented in 
Section 4. In Section 5, we describe two different approaches for deterministic aided STAP and finally, in Section 6, simulations are given to show that the proposed methods outperform the MLED and Stop-Band algorithms.

\section{Data Model}

Consider a radar antenna made of $N$ sensors that acquires $M_{p}$ pulse snapshots for each range gate $l$. We will only use the primary data so we will forget the range gate dimension, also called fast-time dimension. Then, the processing algorithm works independently in each range cell. We adopt the following two hypothesis models where $H_{0}$ and $H_{1}$ mean that no target or a target is present, respectively:

$$
\begin{gathered}
H_{0}: \mathbf{X}=\mathbf{N}, \\
H_{1}: \mathbf{X}=\alpha \mathbf{s}_{s} \mathbf{s}_{t}^{T}+\mathbf{N},
\end{gathered}
$$

where the received data have been arranged into an $M N \times$ $K_{t}$ matrix $\mathbf{X}$ with $K_{t}$ being the number of training data pulse snapshots, $M$ being the number of pulses of the spatio-temporal vector, and $\alpha$ being the complex amplitude of the target. $\mathbf{s}_{s}$ is the spatiotemporal steering vector (length $N M$ ), $\mathbf{s}_{t}$ is the temporal steering vector (length $K_{t}=M_{p}-M+1$ ), and $\mathbf{N}$ is the interference (clutter plus noise) matrix.

We make use of a temporal sliding window to work on the temporal dimension; consequently, the estimated covariance matrix $\mathbf{R}$ is obtained from $\mathbf{X}$ as follows:

$$
\mathbf{R}=\frac{1}{K_{t}} \mathbf{X X}^{H}
$$

One classical STAP detector taken as reference uses the Adaptive Matched Filter (AMF) $[1,2]$. The filter $\mathbf{w}$ is

$$
\mathbf{w}=\frac{\mathbf{R}^{-1} \mathbf{s}}{\mathbf{s}_{s}^{H} \mathbf{R}^{-1} \mathbf{s}_{s}} .
$$

Detection is achieved by comparing the output SNIR power of the matched filter to a threshold as follows:

$$
\mathbf{P}_{\mathrm{AMF}}=\frac{\left|\mathbf{s}_{s}^{H} \mathbf{R}^{-1} \mathbf{X}\right|^{2}}{\mathbf{s}_{s}^{H} \mathbf{R}^{-1} \mathbf{s}_{s}} \underset{H_{1}}{\stackrel{H_{0}}{\lessgtr}} \eta .
$$

In case where a strong target is present at this range gate, $\mathbf{R}$ contains the target covariance matrix. Consequently, the target is removed with the clutter and it can no longer be detected by (4). This happens when many targets are moving at the same speed but are at different distances (roads, highways, convoys, etc.). Another problem with this detector is that the ground clutter has to be homogeneous on the range domain. Otherwise, the clutter used to estimate the covariance matrix will not be representative of the clutter that has to be canceled, leading to a bad clutter rejection.

\section{APES-Based STAP Detectors}

3.1. The Maximum Likelihood Estimation Detector. To overcome the previous issues of signal suppression or the none representativeness of secondary data, the MLED detector [7] based on the APES [8] algorithm removes the signal of interest from the covariance matrix. The problem is stated as follows:

$$
\min _{\mathbf{w}, \alpha}\left(\mathbf{w}^{H} \mathbf{X}-\alpha \mathbf{s}_{t}^{T}\right)\left(\mathbf{w}^{H} \mathbf{X}-\alpha \mathbf{s}_{t}^{T}\right)^{H}, \quad \text { s.t } \mathbf{w}^{H} \mathbf{s}_{s}=1 .
$$

The obtained solution is

$$
\mathbf{w}=\frac{\mathbf{Q}^{-1} \mathbf{s}_{s}}{\mathbf{s}_{s}^{H} \mathbf{Q}^{-1} \mathbf{s}_{s}}, \quad \alpha=\frac{\mathbf{w}^{H} \mathbf{X} \mathbf{s}_{t}^{*}}{K_{t}},
$$

where

$$
\mathbf{Q}=\mathbf{R}-\mathbf{g g}^{\mathbf{H}}, \quad \mathbf{g}=\frac{\mathbf{X s}_{\mathbf{t}}^{*}}{K_{t}} .
$$

Detection is achieved using the output power normalized by the Adaptive Power Residue $\left(\mathrm{APR}=\mathbf{w}^{H} \mathrm{Q} w=\mathbf{s}_{s}^{H} \mathbf{Q}^{-1} \mathbf{s}_{s}\right)$ as follows:

$$
\mathbf{P}_{\text {MLED }}=\frac{\left|\mathbf{s}_{\mathbf{s}}^{\mathbf{H}} \mathbf{Q}^{-1} \mathbf{g}\right|^{2}}{\mathbf{s}_{s}^{H} \mathbf{Q}^{-1} \mathbf{s}_{s}} \underset{H_{1}}{\stackrel{H_{0}}{\lessgtr}} \eta .
$$

To avoid strong signal loss due to covariance matrix estimation errors [9], one may use in addition diagonal loading [10] or subspace methods [11].

3.2. Extension to Stop-Band APES. Because the MLED algorithm is a high-resolution method, it requires an oversampling in Doppler frequency, typically by a factor four, to correctly work. Indeed, combining (5) and (6), it follows

$$
\begin{gathered}
\mathbf{w}^{H}\left(\mathbf{X}-\mathbf{X} \frac{\mathbf{s}_{\mathbf{t}}^{*} \mathbf{s}_{\mathbf{t}}^{\mathbf{T}}}{\mathbf{s}_{t}^{T} \mathbf{s}_{t}^{*}}\right)\left(\mathbf{X}-\mathbf{X} \frac{\mathbf{s}_{\mathbf{t}}^{*} \mathbf{s}_{\mathbf{t}}^{\mathbf{T}}}{\mathbf{s}_{t}^{T} \mathbf{s}_{t}^{*}}\right)^{H} \mathbf{w} \\
=\mathbf{w}^{H} \mathbf{X}\left(\mathbf{I}-\mathbf{P}_{/ /}\right)\left(\mathbf{I}-\mathbf{P}_{/ /}\right)^{H} \mathbf{X} \mathbf{w},
\end{gathered}
$$

where $\mathbf{P}_{/ /}$is the projector into the target signal subspace:

$$
\mathbf{P}_{/ /}=\frac{\mathbf{s}_{\mathbf{t}}^{*} \mathbf{s}_{\mathbf{t}}^{\mathbf{T}}}{\mathbf{s}_{t}^{T} \mathbf{s}_{t}^{*}}=\frac{\mathbf{s}_{\mathbf{t}}^{*} \mathbf{s}_{\mathbf{t}}^{\mathbf{T}}}{K_{t}} .
$$

The problem (5) can then be recognized as a minimization of the interference-plus-noise energy outside the subspace spanned by the target as follows:

$$
\min _{\mathbf{w}}\left\{\mathbf{w}^{\mathbf{H}} \mathbf{X}\left(\mathbf{I}-\mathbf{P}_{/ /}\right)\left(\mathbf{I}-\mathbf{P}_{/ /}\right)^{H} \mathbf{X w}\right\}, \quad \text { s.t } \mathbf{w}^{H} \mathbf{s}_{s}=1 .
$$

The solution is still $\mathbf{w}=\left(\mathbf{Q}^{(-1)} \mathbf{s}_{s}\right) /\left(\mathbf{s}_{s}^{H} \mathbf{Q}^{(-1)} \mathbf{s}_{s}\right)$ but with the more general form for $\mathbf{Q}$ :

$$
\mathbf{Q}=\frac{\mathbf{X} \mathbf{X}^{H}}{\mathbf{s}_{t}^{T} \mathbf{s}_{t}^{*}}-\frac{1}{K_{t}} \mathbf{X} \mathbf{P}_{/ /} \mathbf{X}^{H} .
$$

This latest formulation not only shows the hyperresolution property along the frequency domain but also allows overcoming one major drawback of the MLED method for our application. The MLED has indeed a high-frequency resolution due to the sharpness of the projection $\mathbf{I}-\mathbf{P}_{/ /}$with 


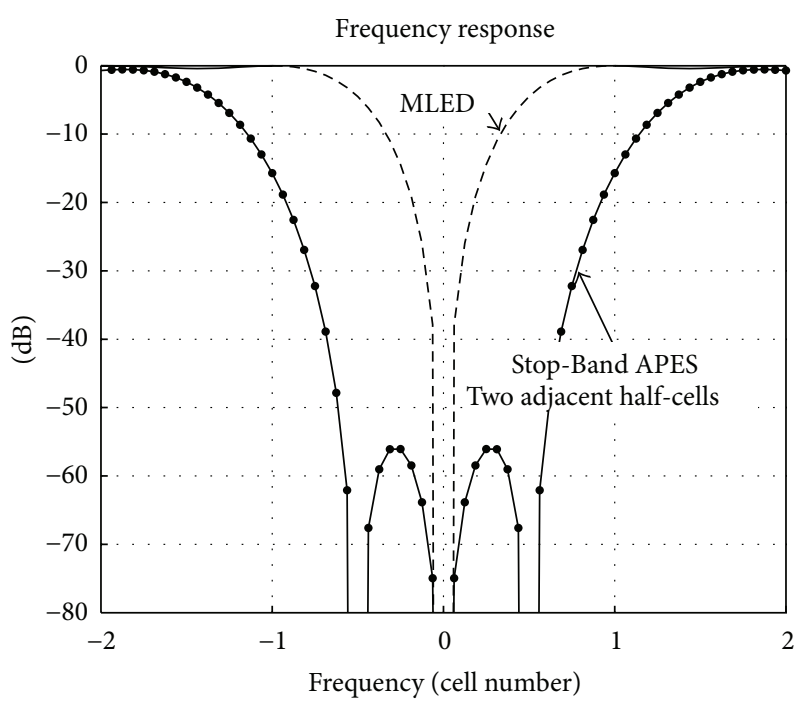

FIGURE 1: Spectral response of regular MLED $\mathbf{S}=\mathbf{s}_{t}$ (dash curve) and Stop-Band APES with two adjacent half-cells (solid curve).

$\mathbf{P}_{/ /}=\left(\mathbf{s}_{\mathbf{t}}^{*} \mathbf{s}_{\mathbf{t}}^{\mathbf{T}}\right) /\left(\mathbf{s}_{t}^{T} \mathbf{s}_{t}^{*}\right)$ (dash curve, Figure 1). This is a problem because it requires a strong oversampling to be sure to remove the signal of interest from the covariance matrix and so it leads to an important increase of the computing load. In order to avoid this problem, we propose a new detector called StopBand APES. The minimization is using a projector $\mathbf{P}_{/ /}$on an extended subspace around the Doppler frequency $f_{0}$ under test. For instance, two adjacent half-cells can be added into the space spanned by $\mathbf{P}_{/ /}=\mathbf{S}_{t}^{*}\left(\mathbf{S}_{t}^{T} \mathbf{S}_{t}^{*}\right)^{-1} \mathbf{S}_{t}^{T}$ with

$$
\mathbf{S}_{t}=\left[\mathbf{s}_{\mathbf{t}}\left(f_{0}-\frac{1}{2 K_{t}}\right), \mathbf{s}_{\mathbf{t}}\left(f_{0}\right), \mathbf{s}_{\mathbf{t}}\left(f_{0}+\frac{1}{2 K_{t}}\right)\right] .
$$

The sharpness and effectiveness of the cancellation around the target signal are characterized by the frequency response of the projector, which is, for a signal $\mathbf{X}$ at frequency $f(\mathbf{X}=$ $\left.\mathbf{s}_{\mathbf{t}}^{\mathrm{T}}(f)\right)$, as follows:

$$
\begin{aligned}
\widetilde{P}_{\perp}(f) & =\left[\mathbf{s}_{\mathbf{t}}^{\mathbf{T}}(f)\left(\mathbf{I d}-\mathbf{P}_{/ /}\right)\right] \frac{\mathbf{s}_{\mathbf{t}}^{*}(f)}{\mathbf{s}_{\mathbf{t}}^{\mathbf{T}}(f) \mathbf{s}_{\mathbf{t}}^{*}(f)} \\
& =1-\mathbf{s}_{\mathbf{t}}^{\mathbf{T}}\left(f_{1}\right) \frac{\mathbf{P}_{/ /}}{K_{T}} \mathbf{s}_{\mathbf{t}}^{*}(f) .
\end{aligned}
$$

Figure 1 shows that building a projector with two adjacent half-cells is enough to correctly remove the signal in the cell under test. Nevertheless, compared to the MLED, the StopBand APES does not require oversampling of the Doppler resolution for the calculation and the application of the STAP filter. A zero-padding by a factor of 2 will still be required to access the signal that has to be evaluated every half-resolution cells for the creation of the projector $[12,13]$.

3.3. Limitations of the MLED and Stop-Band APES. In order to explore the use of subspace-based methods, we have to go deeper in the formulation of the MLED detector. Indeed, these methods will only work if the clutter subspace of

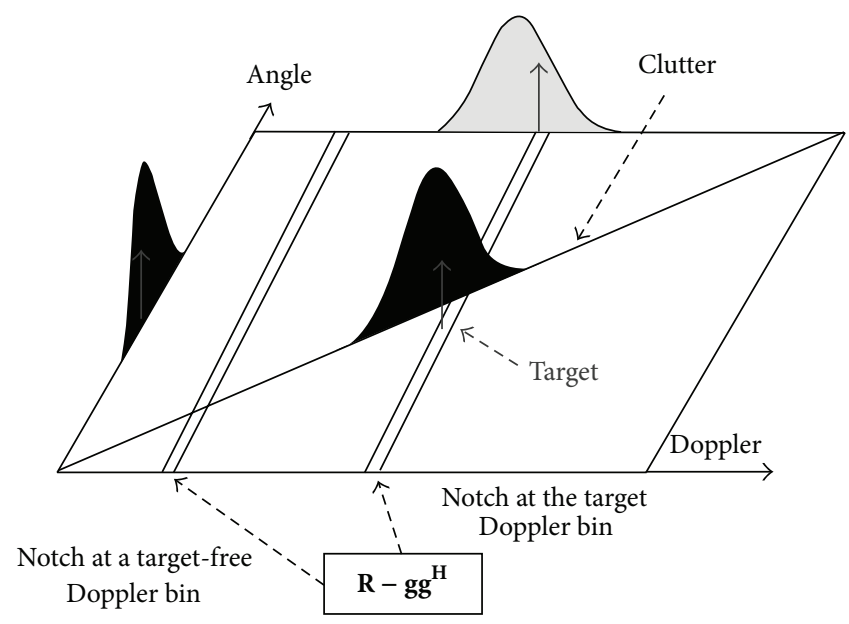

Figure 2: Angle-Doppler map showing the effect of MLED projector for two different Doppler bins.

the covariance matrix $\mathbf{R}$ remains very close to the clutter subspace of the target-free covariance matrix $\mathbf{Q}$. For a given distance cell, if there is no target at this range, the covariance matrix $\mathbf{R}$ only contains interference, that is, clutter and possibly jamming signal and noise, according to (2) as follows:

$$
\mathbf{R}=\frac{\mathbf{X X}^{H}}{K_{t}}=\frac{\mathbf{N N}^{H}}{K_{t}}
$$

We can demonstrate [11] that the matrix $\mathbf{Q}$ is, without approximation:

$$
\mathbf{Q}=\frac{\mathbf{N N}^{\mathrm{H}}}{K_{t}}-\frac{\mathbf{N s}_{t}^{*} \mathbf{s}_{t}^{T} \mathbf{N}^{H}}{K_{t}^{2}} .
$$

The matrix $\left(\mathbf{N N}^{\mathrm{H}}\right) / K_{t}$ is the interference-plus-noise estimated covariance matrix, whereas $\left(\mathbf{N s}_{t}^{*} \mathbf{s}_{t}^{T} \mathbf{N}^{H}\right) /\left(K_{t}^{2}\right)$ is the scalar product of interference-plus-noise vectors with their projection on $\mathbf{s}_{t}^{*}$. It follows from (16) that the modified covariance matrix $\mathbf{Q}$ used for MLED in (8) does no longer contain the target contribution and that the target will not be removed contrarily to the clutter by the MLED STAP filter (6).

The residual clutter-plus-noise covariance matrix is slightly different from the actual covariance matrix $\left(\mathbf{N N}^{H}\right) /$ $K_{t}$ (Figure 2). The term $\left(\mathbf{N s}_{t}^{*} \mathbf{s}_{t}^{\mathrm{T}} \mathbf{N}^{\mathrm{H}}\right) /\left(K_{t}^{2}\right)$ represents the part of the clutter that is removed from the covariance matrix. The number of Doppler cells being usually high, the projector is consequently very sharp; that, the term $\left(\mathbf{N s}_{t}^{*} \mathbf{s}_{\mathbf{t}}^{\mathbf{T}} \mathbf{N}^{\mathbf{H}}\right) /\left(K_{t}^{2}\right)$ is small and both MLED and Stop-Band APES, which removes a wider part of the clutter from the covariance matrix $\left(\mathbf{N} \mathbf{s}_{t}^{*} \mathbf{s}_{\mathbf{t}}^{\mathrm{T}} \mathbf{N}^{\mathrm{H}}\right) /\left(K_{t}^{2}\right)$, are all working. This effect can be seen on Figure 2 in a situation with and without target in the Doppler cell tested.

However, in a situation where the number of Doppler cells is low, we will observe a degradation of the clutter rejection performance of the MLED detector, and this degradation will 
be even worse for the Stop-Band APES algorithm. This effect is due to the partitioning which is done only in time domain. If spatio-temporal partitioning is employed, only a single bin of the angle-Doppler plane is removed but the computational cost would hugely increase because of the angle-Doppler scanning. We will present in the next section a deterministic processing and, in Section 5, a new method that makes use of deterministic processing to solve this problem.

\section{Deterministic Space-Time Processing}

We will here briefly describe a nonadaptive space-time processing which is the basis of the deterministic aided STAP processing we will introduce in the following section. For a side-mounted antenna, the clutter occupies a one-dimension position in the two-dimensional Doppler-angle domain. The clutter Doppler frequency is a function of the receiving angle as follows:

$$
f=\frac{2 V}{\lambda} \sin \theta \Longrightarrow \theta=\sin ^{-1}\left(\frac{\lambda f}{2 V}\right)
$$

with $f$ being the Doppler frequency of the clutter, $\theta$ being the receiving angle, $V$ being the platform speed, and $\lambda$ being the wavelength of the radar frequency. Knowing this relation, we can build a filter that will remove all the signal that is in the 1D-domain driven by (17). The general form of the filter, which will be referred in the following to non-adaptive or deterministic processing, has the same form as AMF in (3) as follows:

$$
\mathbf{w}^{H}=\frac{\mathbf{s}_{\mathbf{s}}^{\mathbf{H}} \mathbf{K}^{-1}}{\mathbf{s}_{s}^{H} \mathbf{K}^{-1} \mathbf{s}_{s}}
$$

but with

$$
\mathbf{K}=\frac{1}{k} \sum_{i=1}^{k} \mathbf{s}_{\mathbf{c}}\left(\theta_{i}\left(f_{i}\right), f_{i}\right) \mathbf{s}_{c}^{H}\left(\theta_{i}\left(f_{i}\right), f_{i}\right)+\mathbf{\Gamma}_{\mathbf{N}},
$$

where $\Gamma_{\mathrm{N}}$ is the true noise covariance matrix (identity matrix in our case), $k$ is the number of mainlobe clutter patches, and $\mathbf{s}_{c}\left(\theta_{i}\left(f_{i}\right), f_{i}\right)$ is the space-time steering vector of angle $\theta_{i}$ and frequency $f_{i}$ obtained with (17). In the same formulation of the filter as MLED and Stop-Band APES in (6), the matrix for each Doppler cell can be written as follows:

$$
\mathbf{K}^{\prime}=\frac{1}{k^{\prime}} \sum_{i=1}^{k^{\prime}} \mathbf{s}_{\mathbf{c}}\left(\theta_{i}\left(f_{i}\right), f_{i}\right) \mathbf{s}_{c}^{H}\left(\theta_{i}\left(f_{i}\right), f_{i}\right)+\mathbf{\Gamma}_{\mathbf{N}},
$$

where the vector $\mathbf{s}_{c}\left(\theta_{i}\left(f_{i}\right), f_{i}\right)$ is the predicted steering vector of the clutter. In this case, to process one Doppler cell, the steering vector $\mathbf{s}_{\mathbf{c}}\left(\theta_{0}\left(f_{0}\right), f_{0}\right)$ of the Doppler cell under test and the two steering vectors $\mathbf{s}_{\mathbf{c}}\left(\theta_{ \pm 1}\left(f_{ \pm 1}\right), f_{ \pm 1}\right)$ from the adjacent Doppler cells are sufficient to correctly remove the clutter. However, the performance of this nonadaptive approach is very limited in practical situations because of the heterogeneity of the clutter (e.g urban or mountainous areas) and because of antenna/receivers calibration errors which make the real steering vector of the antenna slightly different from the actual steering vector used to build the covariance matrix.

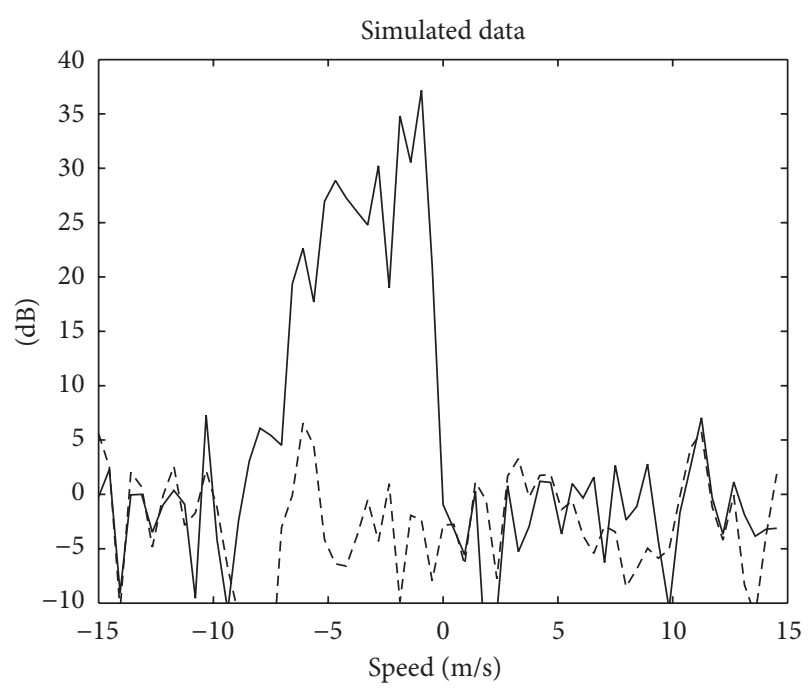

FIGURE 3: Comparison between sum channel (bold curve, negative speeds), deterministic (bold curve, positive speeds), and adaptive space-time processing (dash curve) on error-free simulated data.

To illustrate this effect, we compare the non-adaptive processing (18) to the classic adaptive processing on two sets of data. The first data are the simulated data that we build using the true spatial steering vector and the second set of data is made of realistic data simulated by a STAP simulator that emulates phases errors on the receiving channels and randomly adds impulsive echoes in the clutter. In both cases, clutter is Gaussian, homogeneous, and set to $40 \mathrm{~dB}$. No target is present in these data. A side-looking antenna with four uniformly spaced subarrays is used. Aircraft speed is set to $100 \mathrm{~ms}^{-1}$, radar frequency is $10 \mathrm{GHz}$, and the pulse frequency (PRF) is $2 \mathrm{kHz}$. The non-adaptive processing is only applied in the positive speed domain, that is to say that the negative speeds show the sum channel. The adaptive processing is applied on all the Doppler (speed) domains.

As we can see from Figures 3 and 4, non-adaptive processing works well on the error-free simulated data. The clutterto-noise ratio (CNR) is close to $0 \mathrm{~dB}$, like in the adaptive processing as shown on Figure 5, which implies an attenuation of $40 \mathrm{~dB}$. On the realistic simulated data, the non-adaptive processing is not performing well, as it fails to suppress the clutter. Indeed, as we can see on Figure 6, the residual CNR is near $15 \mathrm{~dB}$ in the main lobe; the clutter attenuation is limited to $25 \mathrm{~dB}$, implying many false alarms. The full range-Doppler maps also point out this effect in Figures 7 and 8. From these results, we deduce that we cannot use a non-adaptive space-time processing in real situations but we may use the deterministic of the clutter Doppler-angle relation together with adaptive processing to achieve better performance.

\section{Deterministic Aided STAP}

5.1. Deterministic Aided GMTI STAP. In GMTI operation, there are two main concerns about heterogeneous environments: clutter heterogeneity (land relief, urban environments) and high-density target area (roads, highways...). 


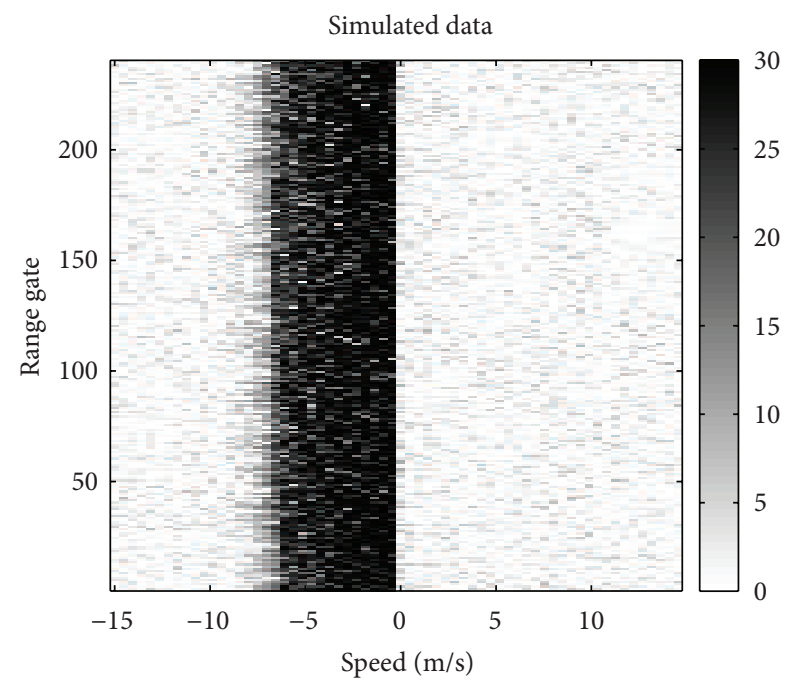

FIGURE 4: Range-Doppler map of the nonadaptive processing (positive speeds) and the sum channel (negative speeds) on error-free data simulated data.

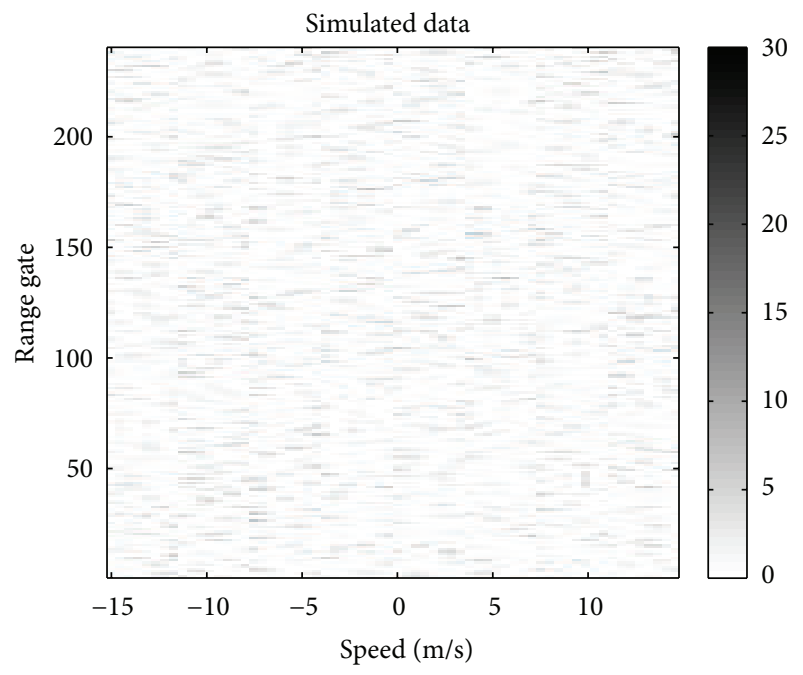

FIGURE 5: Range-Doppler map showing the performance of the adaptive processing on error-free data simulated data.

In many cases, few training data are available and the use of single data set methods is a very helpful alternative (see Section 3.1 and Section 3.2). To overcome the problem of these methods pointed out in Section 3.3, we propose a new method that includes some aspects of the non-adaptive processing. We saw in (16) that the term $\mathbf{g g}^{\mathrm{H}}$ in (7) removes the interest signal (if any) and also a small part of the clutter. The idea here is to try to readd this clutter into the covariance matrix. The covariance matrix is then as follows:

$$
\mathbf{T}=\mathbf{R}-\mathbf{g g}^{\mathrm{H}}+\mathbf{g}_{\mathrm{c}} \mathbf{g}_{\mathrm{c}}^{\mathrm{H}},
$$

where $\mathbf{g}_{\mathbf{c}}$ is the projection of $\mathbf{g}$ on the clutter steering vector $\mathbf{s}_{\mathbf{c}}(\theta(f), f)$ as follows:

$$
\mathbf{g}_{\mathrm{c}}=\mathbf{P}_{\mathrm{c}} \mathbf{g}
$$

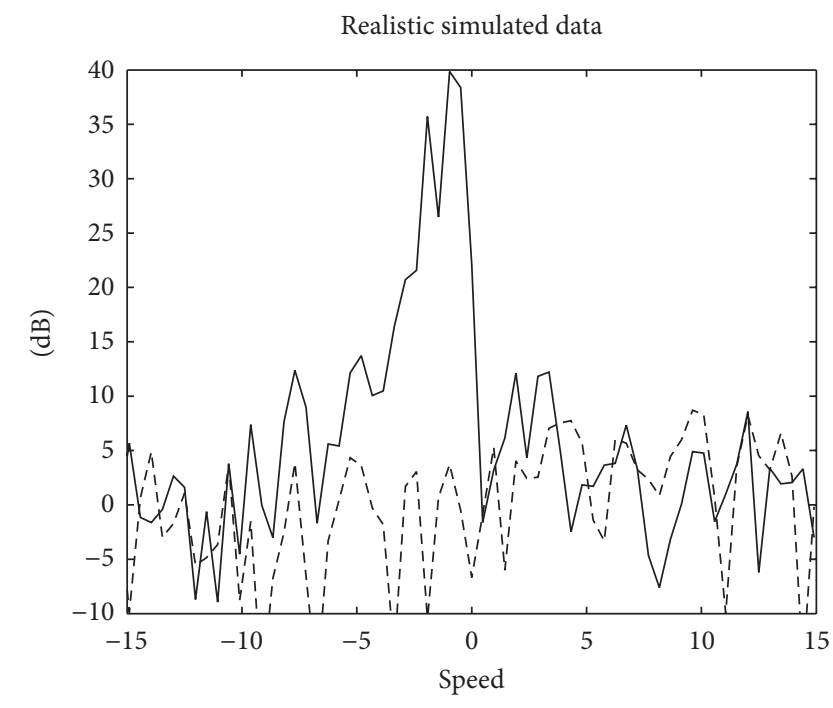

Figure 6: Comparison between sum channel (bold curve, negative speeds), deterministic (bold, positive speeds), and STAP (dash) on realistic data.

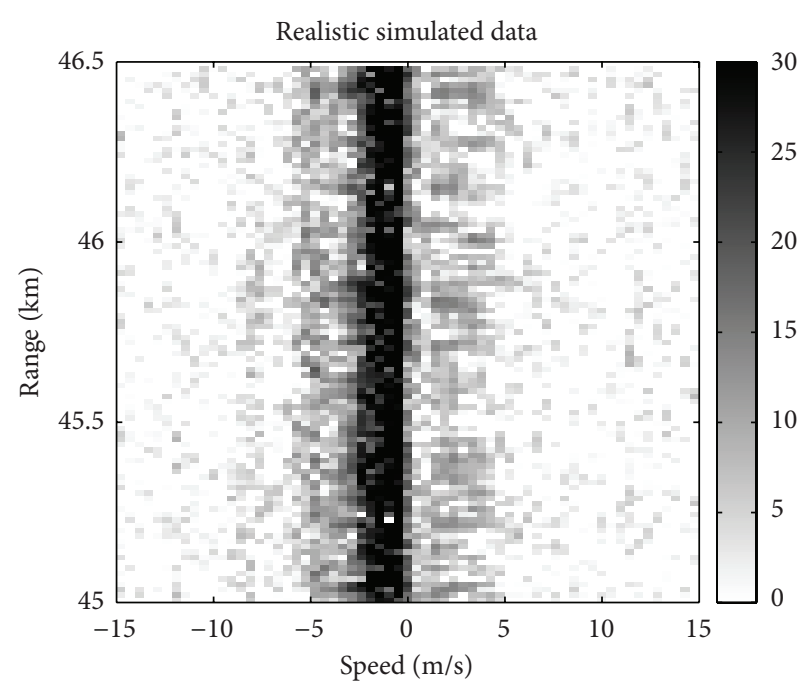

FIGURE 7: Range-Doppler map of the nonadaptive processing (positive speeds) and the sum channel (negative speeds) on realistic data.

with

$$
\mathbf{P}_{c}=\frac{\mathbf{s}_{\mathbf{c}}(\theta(f), f) \mathbf{s}_{\mathbf{c}}^{H}(\theta(f), f)}{\mathbf{s}_{\mathbf{c}}^{H}(\theta(f), f) \mathbf{s}_{\mathbf{c}}(\theta(f), f)} .
$$

We can demonstrate that the covariance matrix $\mathbf{Q}$ of (16) can now be written as follows:

$$
\mathbf{T}=\frac{\mathbf{N N}^{\mathbf{H}}}{K_{t}}-\frac{\mathbf{N s}_{t}^{*} \mathbf{s}_{t}^{T} \mathbf{N}^{H}}{K_{t}^{2}}+\frac{\mathbf{P}_{\mathbf{c}} \mathbf{X} \mathbf{s}_{t}^{*} \mathbf{s}_{t}^{T} \mathbf{X}^{H} \mathbf{P}_{\mathbf{c}}^{\mathbf{H}}}{K_{t}^{2}} .
$$

If the clutter follows the theoretical Doppler-angle relation of (17), then the projection of the signal on the angle-Doppler steering vector will be close to the clutter signal that has been 


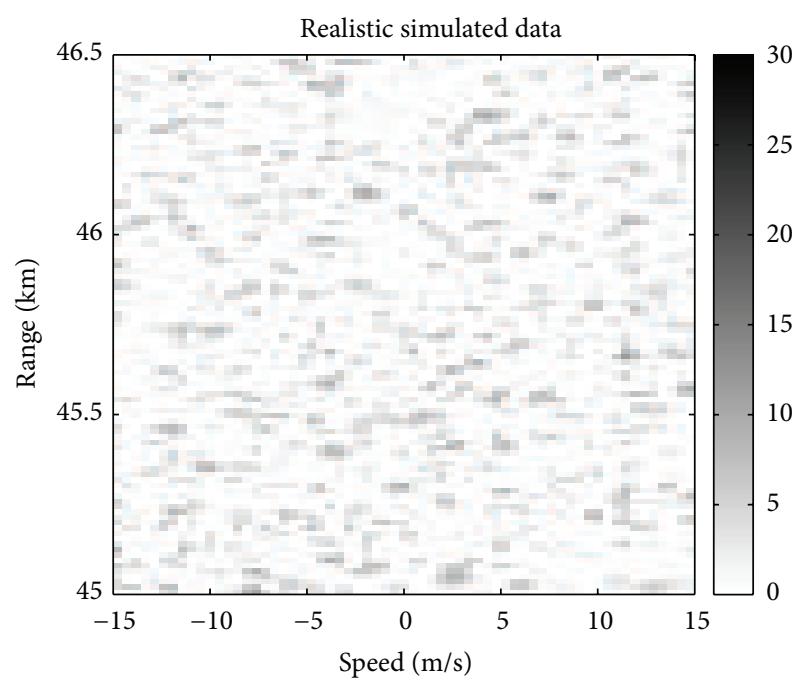

FIGURE 8: Range-Doppler map of the adaptive processing on realistic data.

removed from the matrix $\left(\mathbf{P}_{\mathbf{c}} \mathbf{X} \approx N\right)$, and the covariance matrix $\mathbf{T}$ will be close to

$$
\mathrm{T} \approx \frac{\mathrm{NN}^{\mathrm{H}}}{K_{t}} .
$$

Note that we do not need to set an arbitrary clutter power value because the energy of the clutter is included in $\mathbf{g}_{\mathbf{c}}$ (cf. (22)).

In the case of Stop-Band APES, where the signal notch is wider, we use an extended projector $\mathbf{P}_{\mathbf{c}}$ as follows:

$$
\mathbf{P}_{c}=\frac{\mathbf{S}_{\mathrm{c}} \mathbf{S}_{\mathrm{c}}^{\mathrm{H}}}{\mathbf{S}_{\mathrm{c}}^{\mathrm{H}} \mathbf{S}_{\mathrm{c}}}
$$

with

$$
\mathbf{s}_{c}=\left[\mathbf{s}_{\mathbf{c}}\left(\theta, f-\frac{\Delta f}{2}\right) \mathbf{s}_{\mathbf{c}}(\theta, f) \mathbf{s}_{\mathbf{c}}\left(\theta, f+\frac{\Delta f}{2}\right)\right] .
$$

5.2. Deterministic Aided STAP Processing for Air-to-Air Mode. In air-to-air situations, the problem is different. The spectral occupation of the mainlobe clutter is much smaller than that of GMTI, whereas clutter sidelobes are much more powerful and have to be cancelled. Moreover, target density is very low, compared to GMTI. As we do not have access to a Dopplerangle relation of the mainlobe clutter, we propose another approach to readd this clutter which is partially removed in the APES-based methods. In air-to-air mode, the mainlobe clutter is pretty homogeneous in the range domain.

We will exploit this property to estimate the matrix $\mathbf{g}_{\mathbf{c}} \mathbf{g}_{\mathbf{c}} \mathbf{H}$ on the range gates domain. For each Doppler cell, the covariance matrix $\mathbf{T}$ is defined by

$$
\mathbf{T}=\mathbf{R}-\mathbf{g g}^{\mathrm{H}}+\mathbf{C} \text {. }
$$

TABLE 1: Target position (GMTI data).

\begin{tabular}{lccc}
\hline & Target 1 & Target 2 & Target 3 \\
\hline Speed (m/s) & 3.0 & 5.30 & 5.85 \\
Range (number) & 214 & 138 & 149 \\
\hline
\end{tabular}

However, the matrix $\mathbf{C}$ which was equal to $\mathbf{g}_{\mathbf{c}} \mathbf{g}_{\mathbf{c}}^{\mathbf{H}}$ in (21) is now estimated as follows for each Doppler cells:

$$
\mathbf{C}=\sum_{i=1}^{N} \frac{1}{N} \mathbf{g}_{i} \mathbf{g}_{i}^{\mathbf{H}}
$$

with $\mathbf{g}_{i}$ being the vector $\mathbf{g}=\mathbf{X s}_{t}^{*} / K_{t}$ of the range cell $i$ and $N$ being the total number of range cells. If the clutter is homogeneous, then we can make the following approximation:

$$
\mathbf{C}=\frac{1}{N} \sum_{i=1}^{N} \mathbf{g}_{i} \mathbf{g}_{i}^{\mathbf{H}} \approx \mathbf{g}_{\mathbf{c}} \mathbf{g}_{\mathbf{c}}^{\mathbf{H}} .
$$

This assumption implies that only the homogeneous component of the clutter will be readded in the covariance matrix. The density of the target has to remain low, otherwise useful signal will be nonnegligible in the matrix and SNR of targets will be attenuated. In the case of Stop-Band APES, (12) becomes

$$
\mathbf{T}=\frac{\mathbf{X} \mathbf{X}^{H}}{\mathbf{s}_{t}^{T} \mathbf{s}_{t}^{*}}-\frac{1}{K_{t}} \mathbf{X} \mathbf{P}_{/ /} \mathbf{X}^{H}+\frac{1}{N} \sum_{i=1}^{N} \mathbf{X}_{i} \mathbf{P}_{/ /} \mathbf{X}_{i}^{H},
$$

with $\mathbf{X}$ the data of the range cell under test, $\mathbf{X}_{i}$ the data of the range cell $i$, and $N$ the total number of range cells.

\section{Results}

6.1. GMTI Simulations. We test the GMTI deterministic aided STAP described in Section 5.1 on real airborne data. These data were obtained using the ONERA RAMSES radar system [14], which is a 4-channel ULA antenna. The aircraft speed is $V_{a}=85 \mathrm{~m} \cdot \mathrm{s}^{-1}$, pulse repetition frequency is PRF = $1.5625 \mathrm{kHz}$, the number of range gates is 300 , the number of time taps used to form the space-time data is $M=6$, and the total number of time snapshots (radar pulses) is 64. Three targets are present in the scene (see Table 1).

The Doppler-range of the sum channel (Figure 9) clearly emphasizes the heterogeneous clutter. The next figures present the results for the classical STAP (estimation on 10 range gates with 2 guard cells), MLED STAP, Stop-Band STAP, and deterministic aided Stop-Band STAP (estimation on 3 range gates with no guard cells for all processing). No oversampling is used for the STAP processors (although a $2 \mathrm{x}$ zero-padding is needed to access the data of the half-resolution Doppler cells in the case of Stop-Band) except for the MLED detector, which uses a $4 \mathrm{x}$-oversampling.

The classical STAP processing fails to correctly remove the heterogeneous clutter (Figure 10). The MLED STAP whose signal notch is very sharp also fails to completely remove the clutter. Due to its property of high resolution, the target Doppler extent is very thin and it is difficult to 


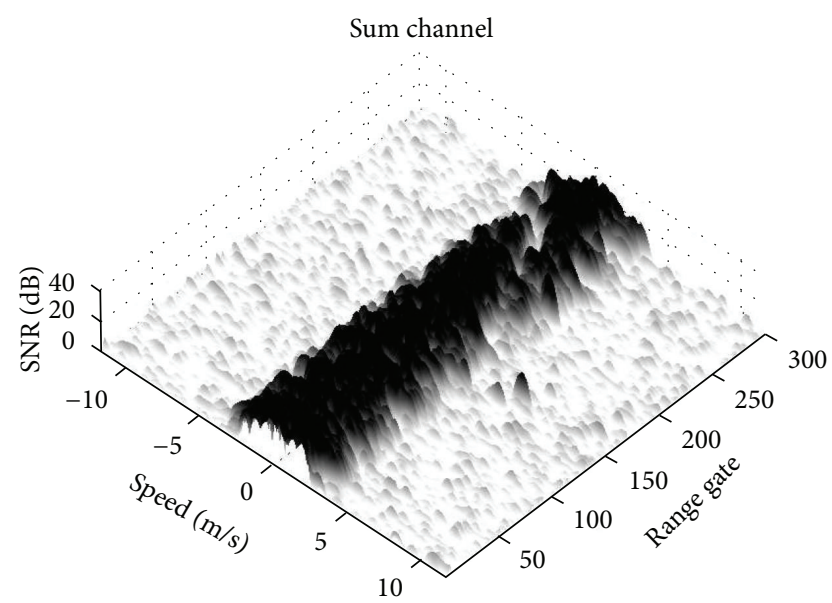

FIGURE 9: Range-Doppler map showing the sum channel of the RAMSES data.

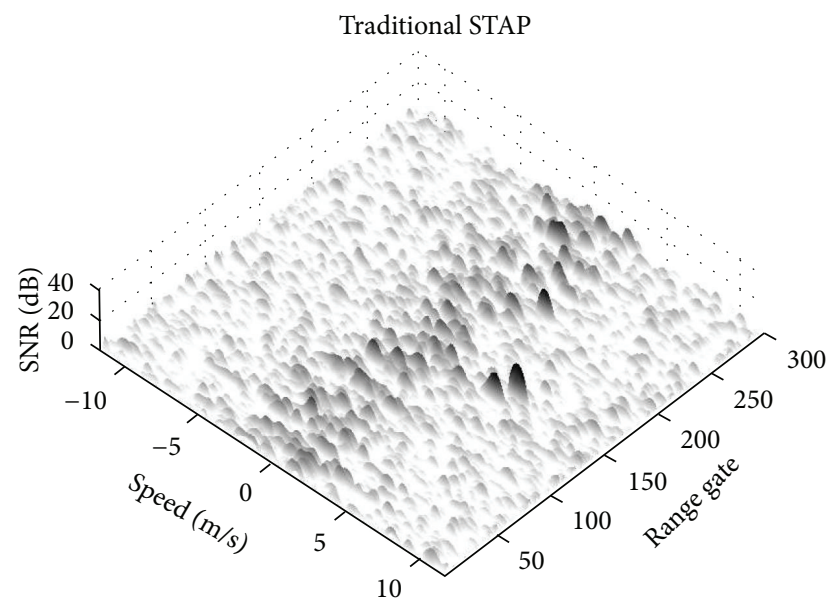

Figure 10: Range-Doppler map on RAMSES data showing the performance of classical STAP processing.

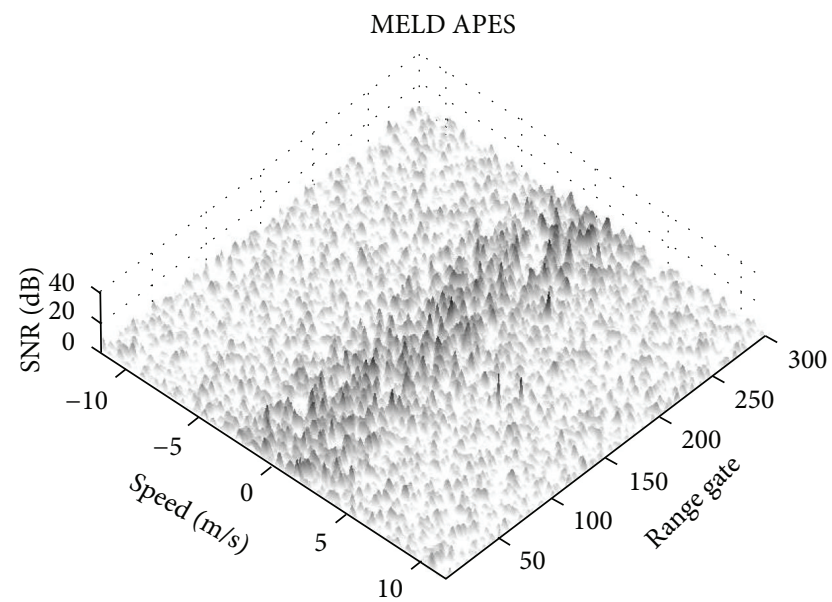

FIGURE 11: Range-Doppler map on RAMSES data showing the performance of the MLED detector.

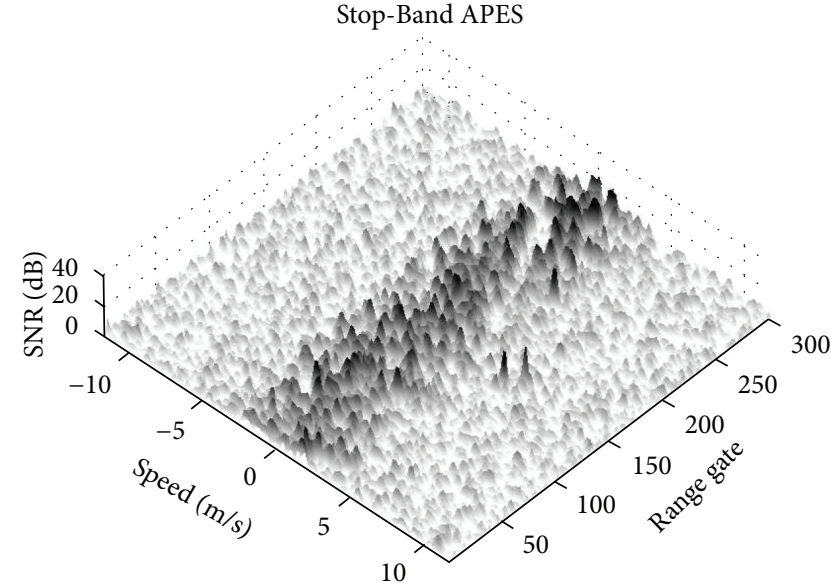

FIGURE 12: Range-Doppler map on RAMSES data showing the performance of the Stop-Band detector.

DA-Stop-Band APES

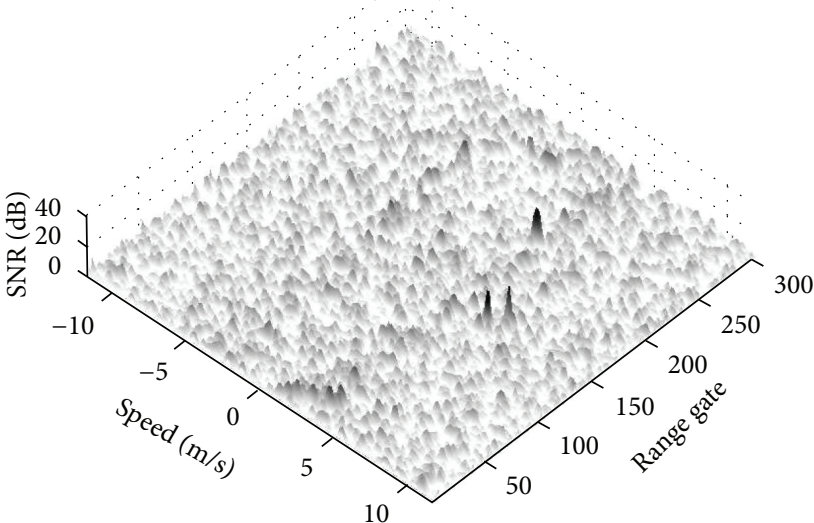

FIGURE 13: Range-Doppler map on RAMSES data showing the performance of the deterministic aidedc Stop Band detector.

distinguish the targets on the range-Doppler map (Figure 11). As predicted, the Stop-Bland STAP processing allows even more clutter to be present as shown in Figure 12, whereas the deterministic aided Stop-Band (DA-Stop-Band) effectively cancels the clutter (Figure 13). This is done without any attenuation on target 1 which lies in the clutter. Figure 14 points out the increased clutter attenuation of DA-Stop-Band over classical Stop-Band for range gate number 149, where target 3 is present. Figure 15 shows the superiority of DA-Stop-Band over classical STAP in clutter rejection for range gate 279 , an area where the clutter is particularly powerful.

6.2. Air-to-Air Simulations. The air-to-air deterministic aided STAP (see Section 5.2) is tested on realistic synthetic data simulating an air-to-air MTI scenario. A front-looking AMSARlike antenna [15] is used for the simulations. The aircraft speed is $V_{a}=300 \mathrm{~m} \cdot \mathrm{s}^{-1}$, pulse repetition frequency is PRF = $20 \mathrm{kHz}$, and the number of range gates is 100 , corresponding to a physical range of $52.5 \mathrm{~km}$ to $59.5 \mathrm{~km}$. The number of time 


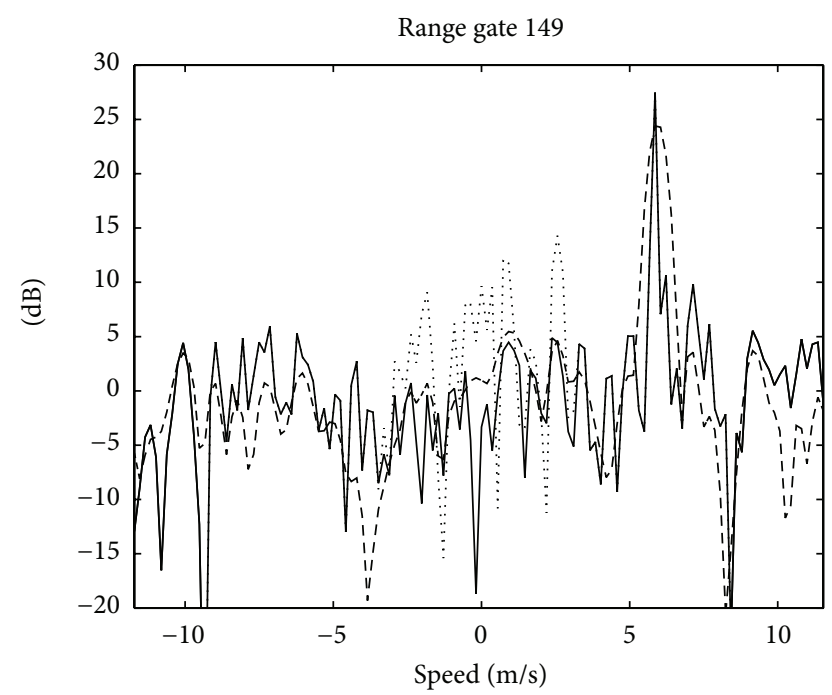

FIGURE 14: Comparison of classical STAP (dash curve) Stop-Band STAP (dot curve) and deterministic aided Stop-Band (solid curve) on RAMSES data for range gate number 149.

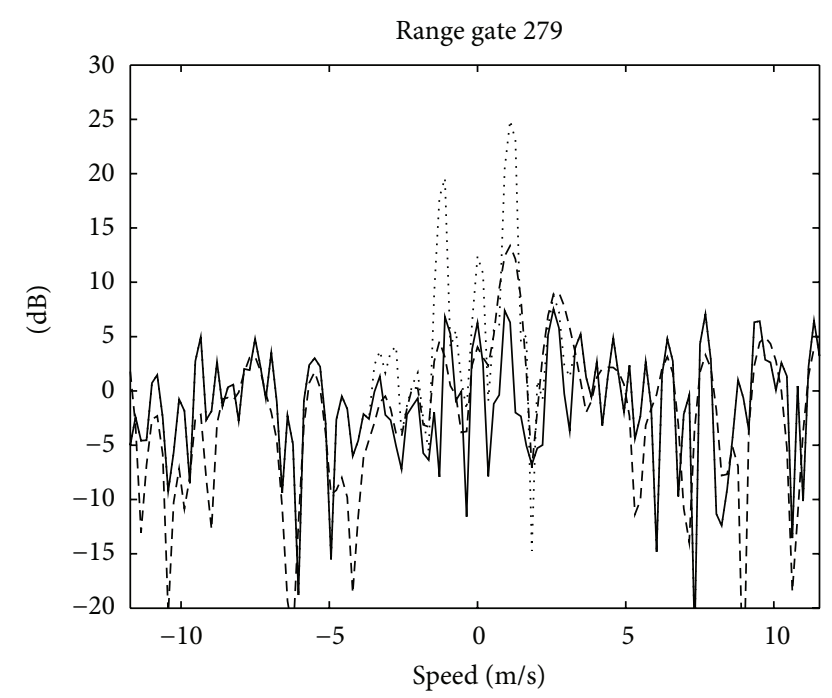

FIGURE 15: Comparison of classical STAP (dash curve) Stop-Band STAP (dot curve) and deterministic aided Stop-Band (solid curve) on RAMSES data for range gate number 279.

taps used to form of space-time data is $K^{\text {taps }}=8$ and the total number of time snapshot (radar pulses) is 128 . Five targets are present in the scene (see Table 2).

The sum channel (Figure 16) clearly shows that the mainlobe clutter (speeds from 230 to $280 \mathrm{~m} / \mathrm{s}$ ) and the sidelobes clutter occupy a wide part of the range-Doppler map. Only two targets on the left-upper part of the map are detectable without STAP processing. On Figure 17, we can see that the classical STAP processing successfully removes the homogeneous main lobe clutter and does not removes the heterogeneous sidelobes clutter. Classical Stop-Band processing cancels almost all the sidelobes clutter but does not suppress
TABLE 2: Target position (air-to-air).

\begin{tabular}{lcc}
\hline Targets & Speed $(\mathrm{m} / \mathrm{s})$ & Range $(\mathrm{km})$ \\
\hline 1 & 50.03 & 58.425 \\
2 & 100.22 & 55.425 \\
3 & 115.026 & 57.00 \\
4 & 185.0265 & 57.30 \\
5 & 216.0296 & 59.475 \\
\hline
\end{tabular}

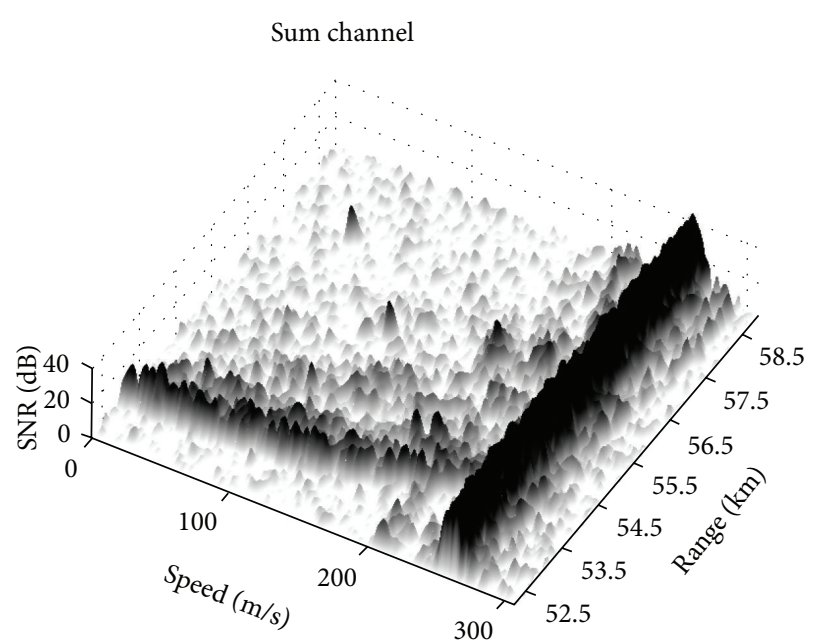

FIGURE 16: Range-Doppler map of the air-air realistic data showing the sum channel.

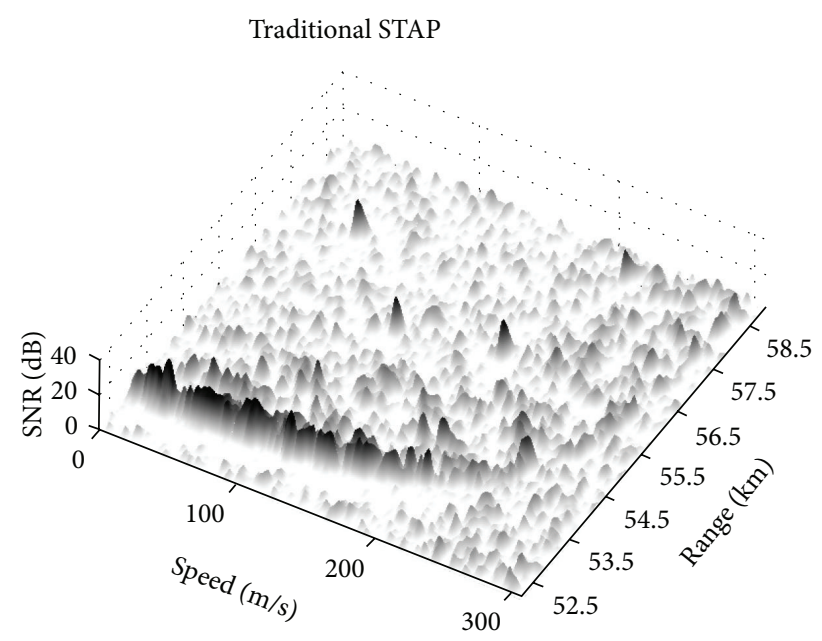

FIGURE 17: Range-Doppler map of the air-air realistic data showing the performance of MLED processing.

all the mainlobe clutter (see Figure 18), whereas DA-StopBand (Figure 19) totally removes it.

On Figure 20, the effect on clutter attenuation of the DAStop-Band is visible through a comparison with classical Stop-Band. We can also observe that both types of Stop-Band processings do not completely remove the sidelobes clutter; this issue can be overcome by using subspace-based algorithms instead of matrix inversion [11]. 


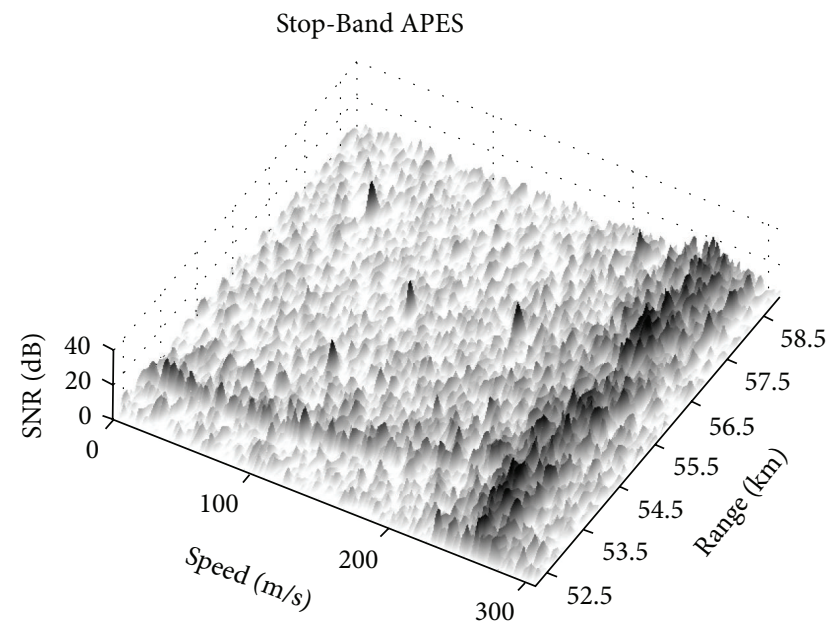

FIGURE 18: Range-Doppler map of the air-air simulated data showing the performance of Stop-Band STAP processing.

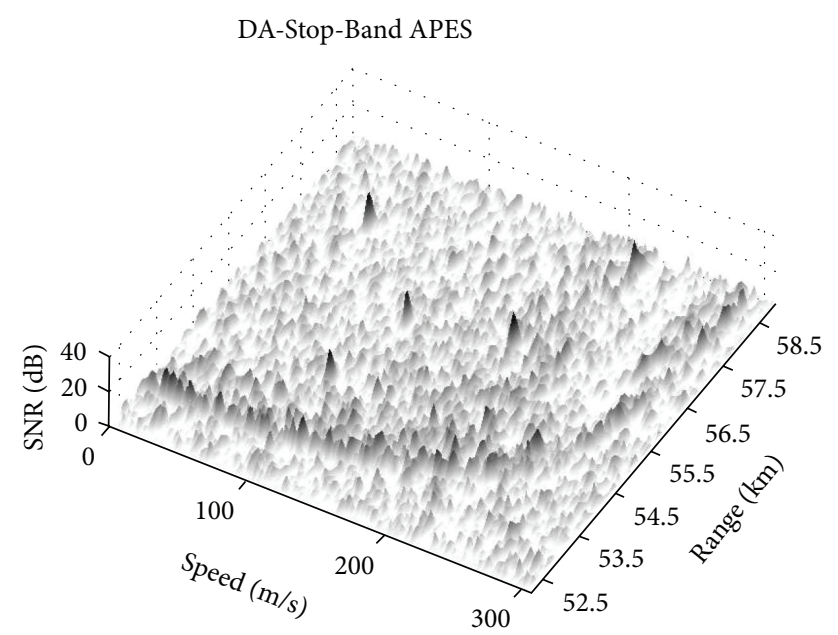

FIGURE 19: Range-Doppler map of the air-air simulated data showing the performance of deterministic aided Stop-Band STAP processing.

\section{Conclusion}

In this paper, we propose two deterministic aided algorithms both based on the APES method. The first algorithm which relies on the deterministic Doppler-angle relation of the clutter is particularly adapted for GMTI detectors. The results on real data show that it outperforms both classical STAP and APES-based algorithms. The second algorithm, which aims to remove the continuous component of the interference, is on the other hand well adapted to air-to-air modes. In this case, the continuous interference is the main lobe clutter. On realistic simulated data, it totally cancels the mainlobe clutter, whereas classical STAP and traditional APES-based algorithms fail, causing many false alarms.

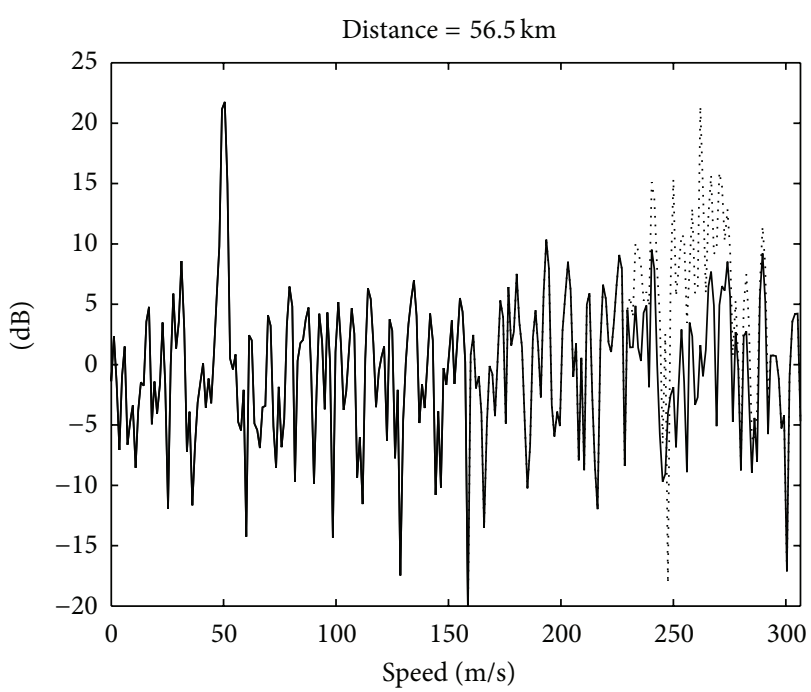

FIGURE 20: Comparison of Stop-Band STAP (dot curve) and Deterministic-Aided Stop-Band (solid curve) on air-air data at a distance of $58.5 \mathrm{~km}$.

\section{Acknowledgment}

The authors would like to thank the DGA from the French Ministry of Defense for their support and funding.

\section{References}

[1] W. L. Melvin, "A STAP overview," IEEE Aerospace and Electronic Systems Magazine, vol. 19, no. 1, pp. 19-35, 2004.

[2] R. Klemm, Principles of Space-Time Adaptive Processing, The Institution of Electrical Engineers (IEE), 2002.

[3] E. Aboutanios and B. Mulgrew, "Evaluation of the single and two data set STAP detection algorithms using measured data," in Proceedings of the IEEE International Geoscience and Remote Sensing Symposium (IGARSS '07), pp. 494-498, June 2007.

[4] P. Wang, H. Li, and B. Himed, "A new parametric GLRT for multichannel adaptive signal detection," IEEE Transactions on Signal Processing, vol. 58, no. 1, pp. 317-325, 2010.

[5] E. Aboutanios and B. Mulgrew, "Hybrid detection approach for STAP in heterogeneous clutter," IEEE Transactions on Aerospace and Electronic Systems, vol. 46, no. 3, pp. 1021-1033, 2010.

[6] M. Zatman, "Properties of Hung-Turner projections and their relationship to the eigencanceller," in Proceedings of the 30th Asilomar Conference on Signals, Systems \& Computers, pp. 11761180, November 1996.

[7] E. Aboutanios and B. Mulgrew, "A stap algorithm for radar target detection in heterogeneous environments," in Proceedings of the IEEE/SP 13th Workshop on Statistical Signal Processing, pp. 966-971, July 2005.

[8] P. Stoica, H. Li, and J. Li, "New derivation of the APES filter," IEEE Signal Processing Letters, vol. 6, no. 8, pp. 205-206, 1999.

[9] L. E. Brennan and L. S. Reed, "Theory of adaptive radar," IEEE Transactions on Aerospace and Electronic Systems, vol. 9, no. 2, pp. 237-252, 1973.

[10] Y. L. Kim, S. U. Pillai, and J. R. Guerci, "Optimal loading factor for minimal sample support space-time adaptive radar," in Proceedings of the IEEE International Conference on Acoustics, 
Speech and Signal Processing (ICASSP '98), vol. 4, pp. 2505-2508, May 1998.

[11] J. F. Degurse, S. Marcos, and L. Savy, "Subspace-based and single dataset methods for STAP in heterogeneous environments," in Proceedings of the IET International Conference on Radar Systems (RADAR '12), pp. 1-6, Glasgow, UK, October 2012.

[12] J.-F. Degurse, L. Savy, R. Perenon, and S. Marcos, "An extended formulation of the maximum likelihood estimation algorithm. Application to space-time adaptive processing," in Proceedings of the International Radar Symposium (IRS '11), pp. 763-768, September 2011.

[13] L. Savy and J. F. Degurse, "Stop-band apes: traitements adaptatifs en environnements heterogenes," Revue Traitement du Signal, vol. 28, pp. 231-256, 2011.

[14] P. Dubois-Fernandez, O. Ruault du Plessis, D. le Coz et al., "The ONERA RAMSES SAR system," in Proceedings of the IEEE International Geoscience and Remote Sensing Symposium (IGARSS '02), vol. 3, pp. 1723-1725, June 2002.

[15] J.-L. Milin, S. Moore, W. Bürger, P.-Y. Triboulloy, M. Royden, and J. Gerster, "AMSAR-a european success story in AESA radar," IEEE Aerospace and Electronic Systems Magazine, vol. 25, no. 2, pp. 21-28, 2010. 

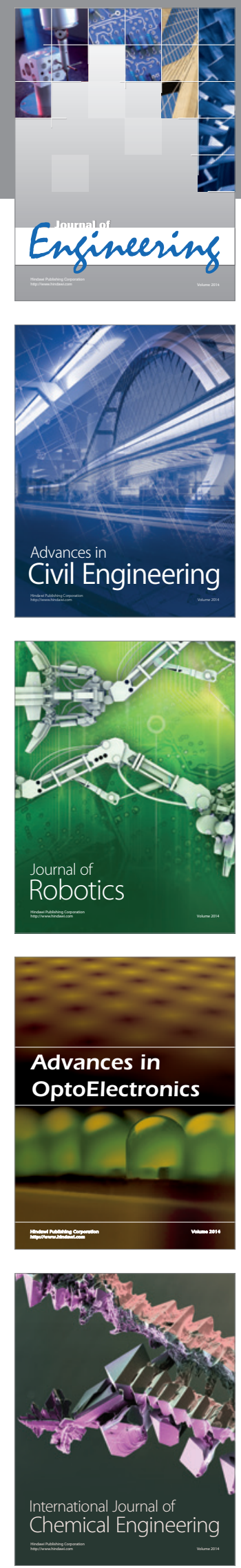

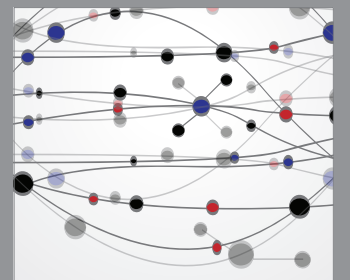

The Scientific World Journal
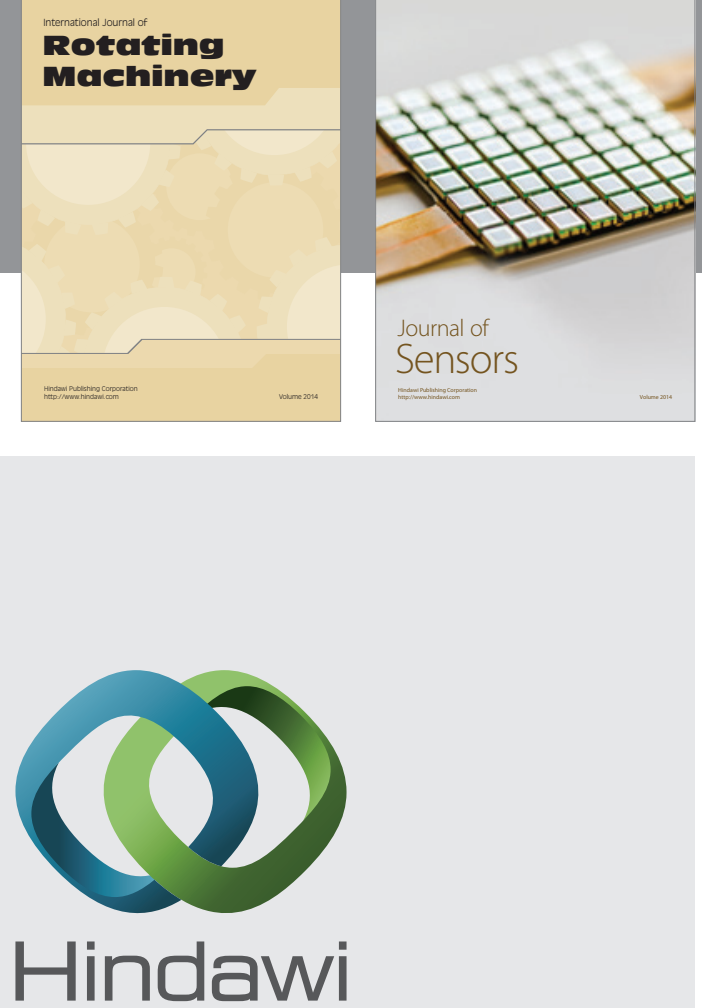

Submit your manuscripts at http://www.hindawi.com
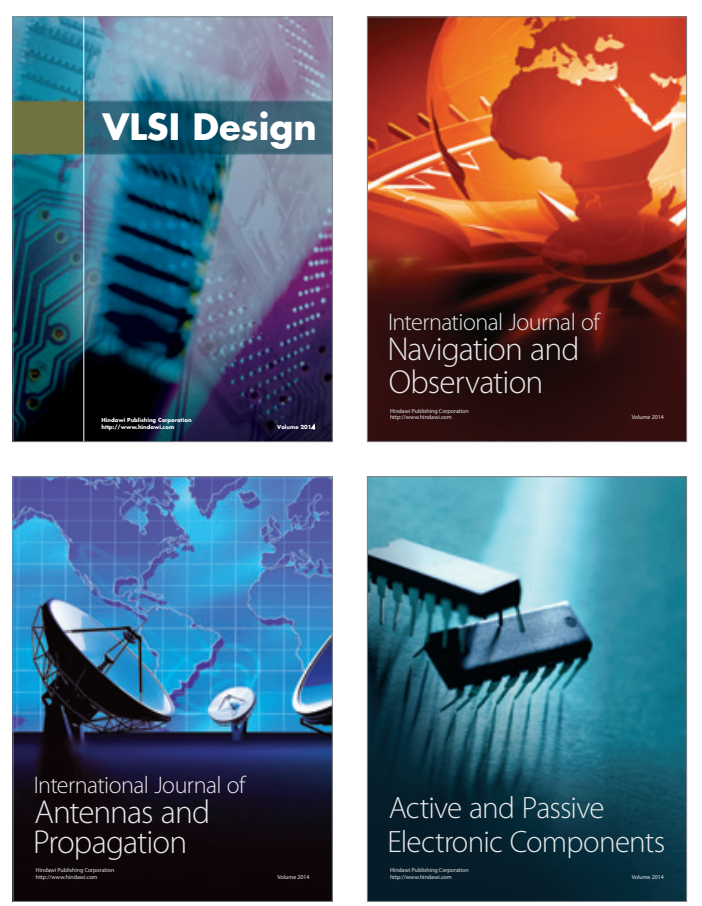
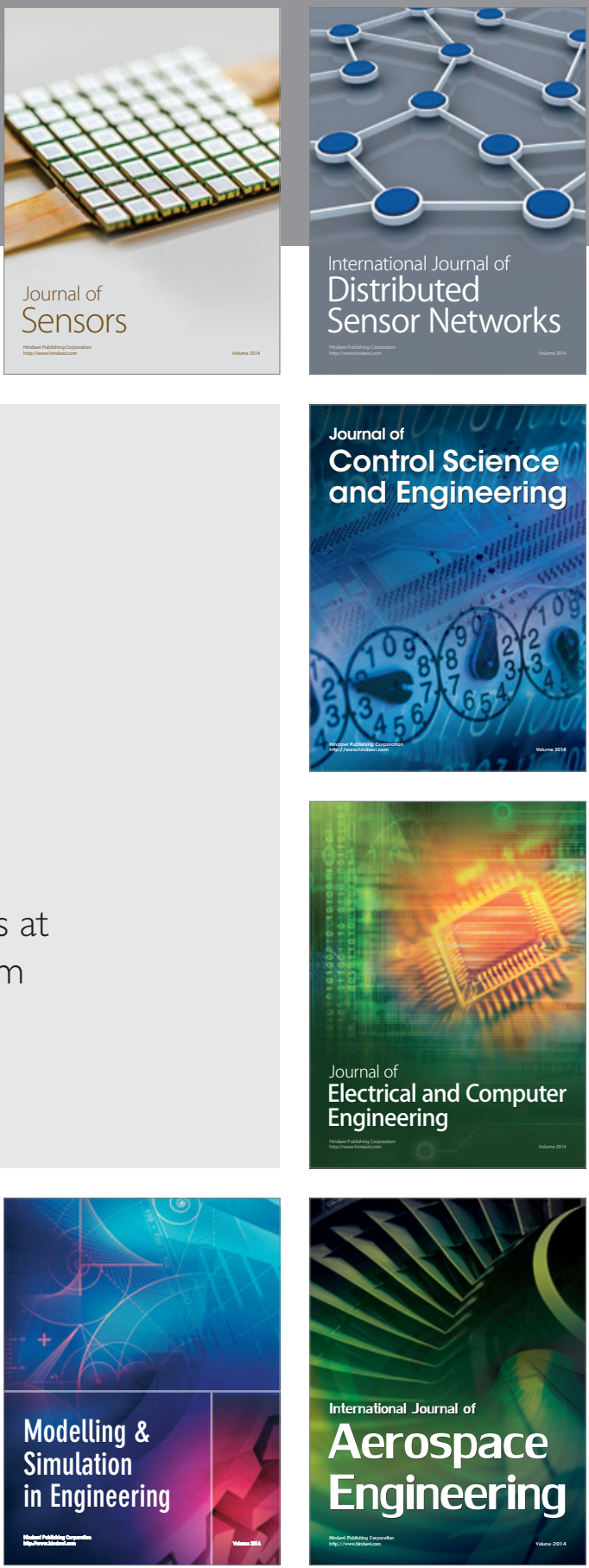

Journal of

Control Science

and Engineering
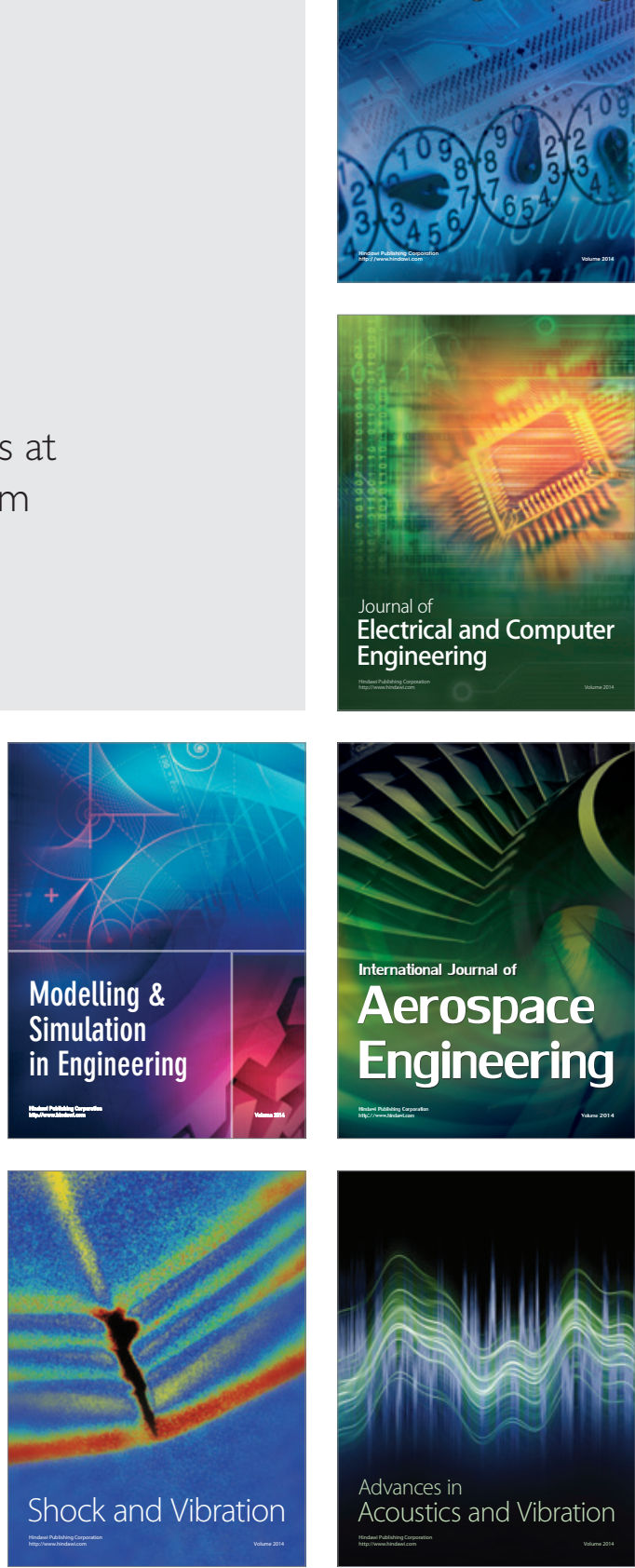